Relations industrielles

Industrial Relations

\title{
Questionnaire d'auto-évaluation du travail en équipe, par Solange Trudeau-Massé. Montréal, Édition du Centre interdisciplinaire de Montréal, Inc., 1971, 26 p.
}

\section{Laurent Bélanger}

\section{Volume 27, numéro 4, 1972}

URI : https://id.erudit.org/iderudit/028344ar

DOI : https://doi.org/10.7202/028344ar

Aller au sommaire du numéro

Éditeur(s)

Département des relations industrielles de l'Université Laval

ISSN

0034-379X (imprimé)

1703-8138 (numérique)

Découvrir la revue

Citer ce compte rendu

Bélanger, L. (1972). Compte rendu de [Questionnaire d'auto-évaluation du travail en équipe, par Solange Trudeau-Massé. Montréal, Édition du Centre interdisciplinaire de Montréal, Inc., 1971, 26 p.] Relations industrielles / Industrial Relations, 27(4), 790-791. https://doi.org/10.7202/028344ar

Tous droits réservés @ Département des relations industrielles de l'Université Laval, 1972
Ce document est protégé par la loi sur le droit d'auteur. L’utilisation des services d’Érudit (y compris la reproduction) est assujettie à sa politique d'utilisation que vous pouvez consulter en ligne.

https://apropos.erudit.org/fr/usagers/politique-dutilisation/ 
portance de l'aspect « qualité 》 du service rendu, l'indivisibilité de nombreux facteurs de production, etc., rendent délicate l'utilisation de l'analyse économique classique dans la recherche du comportement efficace; cela ressort par exemple des travaux de Robert Evans cités par les auteurs. Pourtant ces derniers n'hésitent pas à poser le postulat d'inefficacité et à identifier péremptoirement les coupables (les administrateurs) au chapitre III.

Même le postulat d'absence de souveraineté chez le consommateur n'est pas toujours réaliste. Après tout, le consommateur n'est-il pas souvent en mesure de juger de l'efficacité thérapeutique des services médicaux qui lui ont été rendus? S'il n'est pas satisfait, il peut alors changer de médecin. De plus, les conséquences de ce postulat mentionnées au chapitre I sont aussi, dans le cadre de la médecine libérale, le résultat du paiement à l'acte : conflits d'intérêts, surconsommation, activités préventives réduites, préférence pour les examens superficiels, concurrence médicale, choix du «meilleur 》 traitement et surhospitalisation. L'absence de souveraineté est ni une condition nécessaire ni une condition suffisante dans ces cas.

Plusieurs chapitres de cet ouvrage forment une excellente introduction aux réflexions des économistes face à l'univers de la santé. Les pages à caractère polémique devront toutefois être recommandées avec réserves au lecteur peu averti.

\section{Jean-Yves RIVARD}

\section{Questionnaire d'auto-évaluation du travail}

en équipe, par Solange Trudeau-Masse. Montréal, Editions du Centre interdisciplinaire de Montréal, Inc., 1971, $26 \mathrm{p}$.

Dans tout processus d'apprentissage, la phase d'évaluation demeure importante. Elle l'est d'autant plus lorsqu'elle est effectuée par des gens eux-mêmes impliqués dans l'expérience. Le groupe de travail constitue une de ces expériences. L'évaluation, dans ce cas, est une occasion d'obtenir une information en retour (feedback) sur le fonctionnement même du groupe.

Beaucoup de gens sont réticents à se soumettre à ce genre d'exercice, soit parce qu'ils croient que c'est du narcissisme ou bien une perte de temps; ou encore, soit qu'ils ne savent pas comment procéder. Le fascicule du Centre interdisciplinaire de Montréal présente une technique très simple d'auto-évaluation pour aider ceux qui sont engagés dans des groupes de travail à réfléchir sur leur propre fonctionnement à des moments qu'ils jugent appropriés. Les techniques couramment utilisées dans ce domaine permettent à un groupe de s'évaluer sur les trois dimensions principales du travail d'équipe : la tâche, la procédure et les relations interpersonnelles. Les questionnaires sont construits de façon à dégager une note globale d'efficacité que le groupe peut décomposer pour connaître ses forces et ses faiblesses sur l'une ou l'autre des dimensions. Le questionnaire du CIM diffère passablement de ceux qu'on utilise actuellement, même s'il les recoupe dans leurs dimensions principales. Il est basé sur l'idée fondamentale que le fonctionnement efficace d'un groupe de travail réside dans sa capacité de «faire circuler un degré juste d'informations pertinentes » parmi les membres qui composent le groupe. Les informations couvrent les points suivants :

1. Les objectifs du groupe ou la raison d'être d'une équipe de travail.

2. La sélection des participants.

3. La préparation et le déroulement des réunions.

4. Les modes de participation à un groupe de travail, i.e. l'utilisation et la valorisation des ressources de chacun des membres.

Dans sa forme, le questionnaire fait appel à des questions ouvertes susceptibles de produire des réponses subjectives de la part des membres. Ceci a l'avantage d'inciter le groupe à évoluer à son propre rythme sans être obligé de se comparer à un modèle théorique ou un idéal de fonctionnement efficace. Cet avantage peut se transformer en une lacune lorsque les membres veulent dégager un profil statistique de leur propre fonctionnement en vue de se confronter à un modèle idéal et saisir le chemin qu'ils doivent parcourir pour atteindre un meilleur fonctionnement. En d'autres mots, chaque groupe de travail en utilisant ce questionnaire doit se contenter d'une normalité qui lui est propre sans chercher à savoir si sa performance est meilleure ou pire qu'un autre groupe qui serait confronté à une même situation et éprouverait des difficultés similaires au plan de l'affectivité, de la tâche et de la 
procédure de travail. C'est donc un instrument essentiellement 《didactique », façonné pour une fin d'apprentissage, et non un instrument de recherche empirique. J'aurais aimé discuter de la valeur pratique du questionnaire, mais je n'ai pas eu l'occasion de l'utiliser.

\section{Laurent BELANGER}

Economic Theory of Teams, by Jacob Marschak and Roy Radner, New Haven and London, Yale University Press, 1972, X, 345 p.

Marschak est le père de la théorie économique des «équipes 》 (teams). Avec l'aide de Radner il nous présente une espèce de somme théorique qui n'a plus l'ingéniosité succinte de ses premiers articles sur le sujet. L'intérêt se perd dans toute la partie centrale, excessivement formelle, de ce gros livre. Les premiers chapitres et les derniers stimulent un peu plus le lecteur.

La problématique des équipes est posée de façon simple dès la préface. Ce sont des organisations dont les membres partagent les mêmes intérêts et les mêmes croyances (beliefs), mais n'ont pas le même degré d'information. Le problème qui se pose à ce type d'organisation, ou à un «organisateur » d'équipe peut alors être formulé ainsi : comment répartir les tâches de recherche, de communication et de décision parmi les membres, de façon à atteindre des résultats optimaux, du point de vue de l'organisation ou de celui de l'organisateur. Comme on le voit la perspective est celle de l'économique, définie au sens large comme la science de l'utilisation efficace, ou optimale, de ressources rares.

Dans ce qu'ils croient être une démarche de bonne méthode les auteurs commencent par s'interroger sur la décision individuelle. C'est l'occasion pour eux de présenter de façon cohérente un ensemble de concepts qui seront ensuite utilisés dans l'étude des équipes. Dès ce moment la discussion se perd trop souvent dans une formalisation excessive dont on ne voit pas trop bien l'utilité pour l'étude des problèmes précis qui ont été présentés au début du livre.

La deuxième partie est consacrée à l'étude de ces problèmes qui se posent aux équipes. On passe donc de l'individu à l'organisation, et des concepts à leur application. Comme on l'a déjà signalé, ces applications demeurent excessivement formelles, et surtout elles semblent commandées davantage par les exigences mêmes de la formalisation plutôt que par le souci de répondre à des problèmes concrets. Cette théorie des équipes court ainsi le risque de répéter l'échec de la théorie mathématique des jeux : à appliquer une théorie élaborée selon les seules exigences de la formulation mathématique on n'arrive pas à constituer vraiment les phénomènes sociaux étudiés, c'est-àdire qu'on ne fait pas de science sociale.

Toutefois les auteurs ouvrent dans les chapitres 8 et 9 une voie nouvelle, où leur analyse évite un peu mieux l'écueil qu'on vient de signaler. Ils considèrent le problème d'une équipe comme un problème de réseau de communication qu'il s'agit d'optimaliser. On pourrait croire qu'ils vont rejoindre ainsi les nombreux travaux des psychologues sociaux sur les réseaux de communication. Mais il n'en est rien. Tout au plus s'inspirent-ils des ingénieurs en communication. Pourtant on n'a qu'à lire l'excellent petit livre de Flament Réseaux de communication et structures de groupe, Paris, Dunod, 1965, qui, soit dit en passant, est aussi bon mathématicien que Marschak et Radner, pour voir tout ce que les psychologues sociaux peuvent apporter à une théorie, même économique, des réseaux.

Le spécialiste des relations industrielles ne pourra donc rien tirer de ce livre, à moins qu'il le prenne comme un divertissement mathématique. La théorie économique des vraies équipes, de négociateurs, de permanents syndicaux, de direction des ministères, etc., reste encore à construire.

Vincent LEMIEUX

The Imperfect Union, A History of Corruption in American Trade Unions, by John Hutchinsen, New York, E. P. Dutton \& Co. Inc., 1972, 477 pp.

Cet ouvrage retrace l'histoire de la corruption dans le syndicalisme américain à partir de 1890 jusqu'aux années soixante. Il analyse des cas, essaie d'expliquer l'origine de ce problème, ses causes et sa persistance.

La corruption syndicale est une question complexe pour laquelle il est assez facile de se laisser aller à des jugements subjectifs. Voulant éviter ce piège, l'auteur s'est arrêté à un critère: "Trade unionism should not be employed as an 